\title{
Considerably Enhanced Second-Harmonic Generation in Resonant U-Shaped Nano-Structures
}

\author{
Rana Asgari Sabet and Habib Khoshsima \\ Research Institute for Applied Physics and Astronomy, University of Tabriz, Tabriz, Iran \\ Corresponding author Email: khoshsima@tabrizu.ac.ir
}

Received: Sep. 17, 2017, Revised: Nov. 24, 2017, Accepted: Dec. 23, 2017, Available Online: June. 30,2019

DOI: 10.29252 /ijop.13.1.13

\begin{abstract}
In this paper, we perform a detailed study of the spectral response of the gold U-shaped nano-structures for different geometrical parameters and polarizations in order to obtain significant localization factor in the wavelength $1.55 \mu \mathrm{m}$. The obtained near-field distribution of electric fields reveals that resonances in these nano-structures correspond to the even and odd plasmonic modes depending on the geometrical parameters and polarization directions. Considerably large localization factor is obtained for the first odd mode in specific geometrical parameters. Then, this structure is considered to be surrounded by a typical secondorder nonlinear dielectric. The effective susceptibility is calculated for the considered structure, using the nonlinear retrieval method, to demonstrate the enhanced second-harmonic generation quantitatively. In order to represent the applicability of the investigated structure in nano-scale light sources and frequency doublers, its second harmonic generation efficiency is compared with the efficiency of the nonlinear dielectric alone with the same dimensions.
\end{abstract}

KEYWORDS: Plasmonic modes, localization factor, effective susceptibility, enhanced nonlinearity

\section{I.INTRODUCTION}

Artificial sub-wavelength shaped structures are designed to influence the propagation characterization of electromagnetic fields. Among different configurations, U-shaped structures have attracted special attention of researchers in the past decade[1]-[4]. These structures are simplified configuration of single split-ring resonators (SRRs), which are essential building blocks of metamaterials in microwave and few $\mathrm{THz}$ frequencies [5]. In the microwave part of the spectrum these structures are usually made of copper and can be considered as perfect conductors because the skin depth is much smaller than the metallic feature size [6]. The strong geometric LC resonance in SRRs leads to the negative effective permeability[7]. In order to increase the resonance frequency, these structures were scaled down and negative magnetic response was extended to the mid-infrared spectral range [2], [8]. Since size of the thin sub-wavelength U-shaped components becomes less than the metal skin depth in the optical and near-infrared frequencies, surface plasmon resonances can be excited in these structures. Studying the spectral response of the U-shaped resonators in the near infrared frequencies at normal incidence has been revealed that all resonances are different order plasmonic resonances of the entire structure[9]. It is also demonstrated that for electric field parallel with the gap, plasmonic modes have an odd symmetry, whereas the modes possess even symmetry for the polarization perpendicular to the gap[10]. In another configuration, in which magnetic field is perpendicular to the plane of U-shaped nanostrips, negative refractive index has been obtained in higher order plasmonic modes [11]. Besides, plasmonic resonances of these structures are utilized for other kind of applications such as switches for optical information processing[12]. Strong confinement of the electromagnetic fields on the nano-scale is another feature of the plasmonic resonant structures which allow significant enhancement of weak nonlinear processes[13], [14]. This can be applicable for increasing the nonlinearity of the metal itself or 
of a material adjacent to the metal[14]-[16]. In this regard second harmonic generation (SHG) and third harmonic generation (THG) from the SRRs, due to the broken inversion symmetry of metal, have been investigated theoretically[17], [18] and shown experimentally using femtosecond Gaussian pulses[19]-[21]. Also, the experimental results of SHG from SRRs on GaAs substrate reveal that obtained SHG signals are stronger compared to those from SRRs on the glass substrate [22]. In general, for metallic nano-structures on top of the nonlinear media, the bulk nonlinearity tends to be more dominant than the effect of surface nonlinearity [23]. So it can be inferred that enhancement of nonlinear response of nonlinear dielectrics surrounding the metallic structures is more significant. Therefore, in this paper we intend to show enhancement of the nonlinear response of a typical second-order nonlinear dielectric medium surrounding the SRRs quantitatively. For this purpose, we obtain the near-field distribution of the plasmon modes of gold SRRs for different geometrical parameters. Three even and four odd plasmonic modes are recognized for different sizes of SRRs and different polarization directions in normal incident. This detailed geometrical analysis is done to obtain significant localization factor (LF) in the wavelength $1.55 \mu \mathrm{m}$. Then the SRR with resonance in this wavelength surrounded with nonlinear dielectric is considered as an effective nonlinear medium and its effective second order susceptibility is obtained using the nonlinear retrieval method. The considered structure in our investigation can be achieved using nonlinear substrate and super-states. The obtained results represent that these structures can be considered as efficient frequency converters in nano-scale.

\section{Materials ANd Methods}

For the unit cell of the considered structure the defined geometrical parameters are shown in the Fig. 1.

The selected amounts of these parameters are according to the amounts of the previously done work by Rockstuhl et al. [9] . Thickness $(t)$ and width of the wires $(w)$ are set to the constant values $20 \mathrm{~nm}$ and $60 \mathrm{~nm}$, respectively. The spectral response of the all structures over the frequency range $(30-500 \mathrm{THz})$ is obtained for the electric fields parallel to the $\mathrm{x}$ and $\mathrm{y}$ directions. The relative permittivity of bulk gold is considered the Drude model with plasma frequency $\omega_{p}=1.37 \times 10^{16}\left[\mathrm{~s}^{-1}\right]$ and damping frequency $\omega_{c}=4.08 \times 10^{13}\left[\mathrm{~s}^{-1}\right]$ in the numerical simulations. It has been shown that for the thin gold films considering the damping frequency 1.65 times larger than the bulk value yields good agreement between simulation and experiments[2].

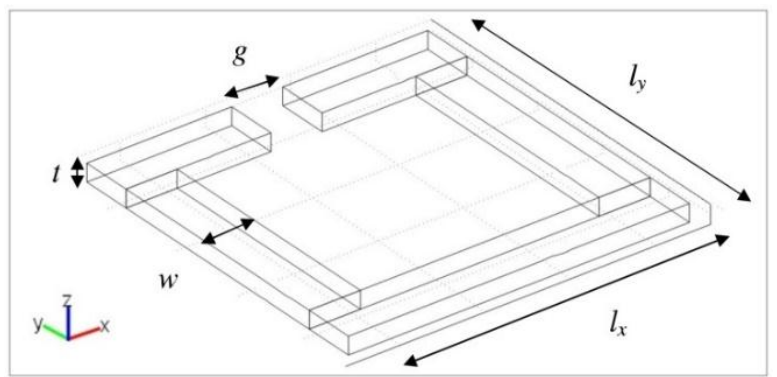

Fig. 1. Schematic sketch of the considered structure with defined geometrical parameters.

To obtain the electromagnetic field distribution and also extraction of the complex scattering parameters for different frequencies, the section "harmonic propagation of the electromagnetic waves" of the Multiphysics simulation software is used. This software solves the partial differential equations with finite element method. Plane wave propagating in the $\mathrm{z}$ direction, is applied to the input port. Perfect electric conductor and perfect magnetic conductor boundary conditions are applied to the boundaries for which the electric field or magnetic field is normal, respectively. These boundary conditions imply the infinite extension of the unit cell in the x-y plane with periods $a_{x}$ and $a_{y}$ in the $\mathrm{x}$ and $\mathrm{y}$ directions, respectively[24], [25]. Second-order LF, $L\left(|E|^{2}\right)$, is introduced as the volume integral of the squared fundamental electric field norm over the considered unit cell divided by the same integral for a homogeneous slab with equivalent linear properties, to measure the degree of field localization[26]. This factor is calculated for all structures using the obtained electric field distribution over the unit cell. 
Then the structure with geometrical parameters, in which the LF is largest in the wavelength $1.55 \mu \mathrm{m}$, is considered to be surrounded by a second order nonlinear dielectric. The susceptibility for the typical nonlinear medium is considered to be $\chi^{(2)}=10^{-12}[\mathrm{~m} / \mathrm{V}]$ [24], [27]. Time domain simulations are performed to obtain the nonlinear fields generated from the metallic SRR embedded in nonlinear dielectric. In time domain simulations the equation for the electromagnetic field is:

$\nabla \times\left(\frac{1}{\mu_{0}} \nabla \times \mathbf{A}\right)+\varepsilon_{r} \varepsilon_{0} \frac{\partial^{2} \mathbf{A}}{\partial t^{2}}=\frac{\partial \mathbf{P}}{\partial t}$

in which, $\mathbf{A}$ is the electromagnetic vector potential and $\mathbf{P}$ is a polarization vector. Equation below is considered for describing the polarization vector in a metal with Drude response[28]

$$
\frac{\partial \mathbf{P}}{\partial t}+\omega_{c} \mathbf{P}=-\varepsilon_{0} \omega_{p}^{2} \mathbf{A}
$$

A time varying plane waves with the wavelength of $1.55 \mu \mathrm{m}$ (corresponding to the resonance frequency, $f=193 \mathrm{THz}$ ) and with the amplitudes $10(\mathrm{GV} / \mathrm{m})$ is applied to the considered structure in a specific time interval. The obtained distribution of the fundamental field is used to calculate the nonlinear polarization in the dielectric medium. Calculated time varying transmitted and reflected signals are Fourier transformed to obtain the complex value of the generated nonlinear field's amplitudes. Then, the effective susceptibility can be retrieved using nonlinear retrieval method. Similar to the retrieve of the linear effective homogenized constitutive parameters of metamaterials[29], homogenized properties can be assigned to the inhomogeneous metallic structures embedded in nonlinear dielectrics[24]. The nonlinear retrieval method is established by Larouche and Smith, in which the known linear effective parameters, sample thickness, input field and the nonlinear transmitted and reflected fields are used to retrieve the effective susceptibility[24], [26]. The nonlinear generated fields can be determined using the experimental or simulation results.

\section{III.RESULTS AND DISCUSSION}

At the first step, geometrical parameters are considered $a_{x}=a_{y}=a=500 \mathrm{~nm}, l_{x}=l_{y}=400 \mathrm{~nm}$ and the gap size is considered $g=0,60,240 \mathrm{~nm}$. Spectral responses of three different structures are obtained and different peaks in the reflectance spectra indicate the occurrence of the resonances. Second-order LF for these structures and two different polarizations are illustrated in Fig. 2.

In the case of closed ring $(\mathrm{g}=0)$, which is identical for the both polarizations, two resonances occur and approximately large LF is obtained in the higher resonance frequency. These resonances are different order plasmonic modes of the entire wire. For the gap size equal to $60 \mathrm{~nm}$, four resonances occur for the $\mathrm{x}$ polarized electric field.

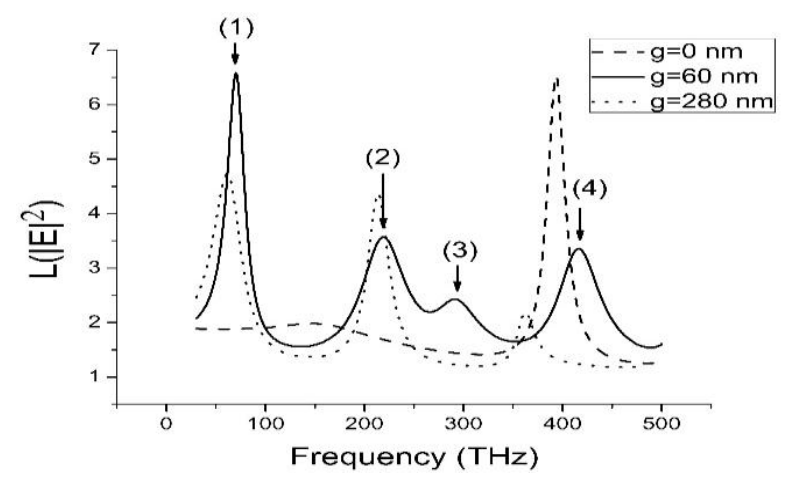

(a)

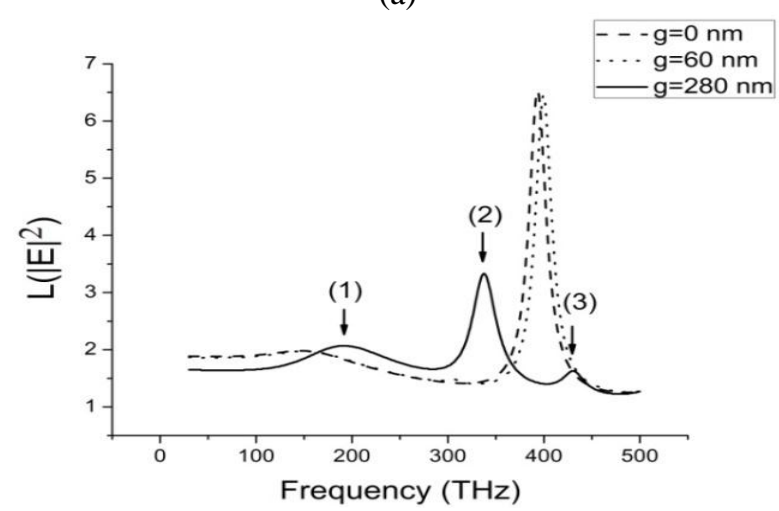

(b)

Fig. 2. Second-order LF for different gap sizes and electric fields parallel to the (a) $\mathrm{x}$ and (b) y direction.

Obtaining the electric field distribution in the resonance frequencies reveals that odd numbers of nodes appear in the near-field distribution. Normalized electric field $\mathrm{z}$ component distribution, at $10 \mathrm{~nm}$ above the structure, for 
these four resonances corresponding to the peaks (1) to (4) in Fig. 2(a), are illustrated in Fig. 3(a-d), respectively. These modes are commonly labelled according to the number of the nodes in field distribution. Occurrence of odd modes can be explained considering the orientation of the structure with respect to the electric field direction.

The electric field in the $\mathrm{x}$ direction causes electric charge oscillations in two legs parallel to the y direction to be in opposite directions. So, the electric fields in these two legs have phase difference of the $\pi[10]$.

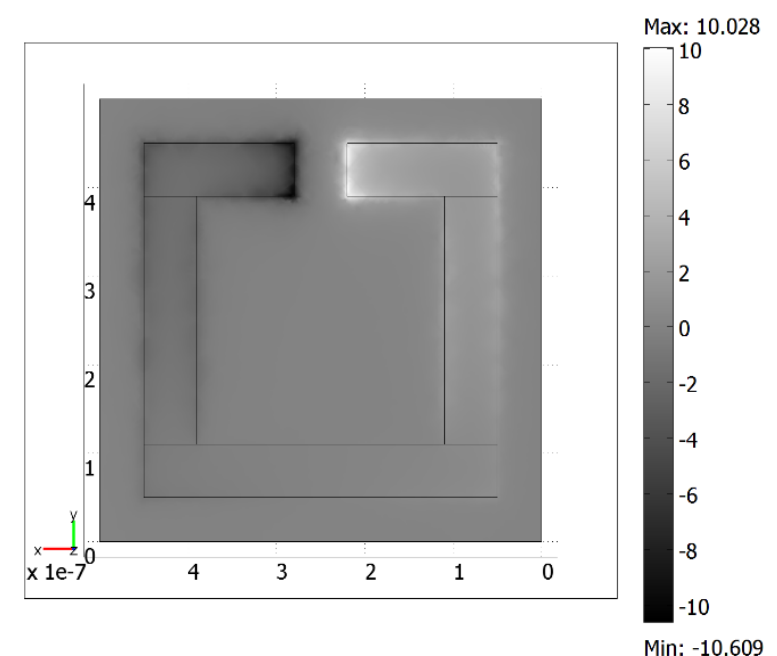

(a)

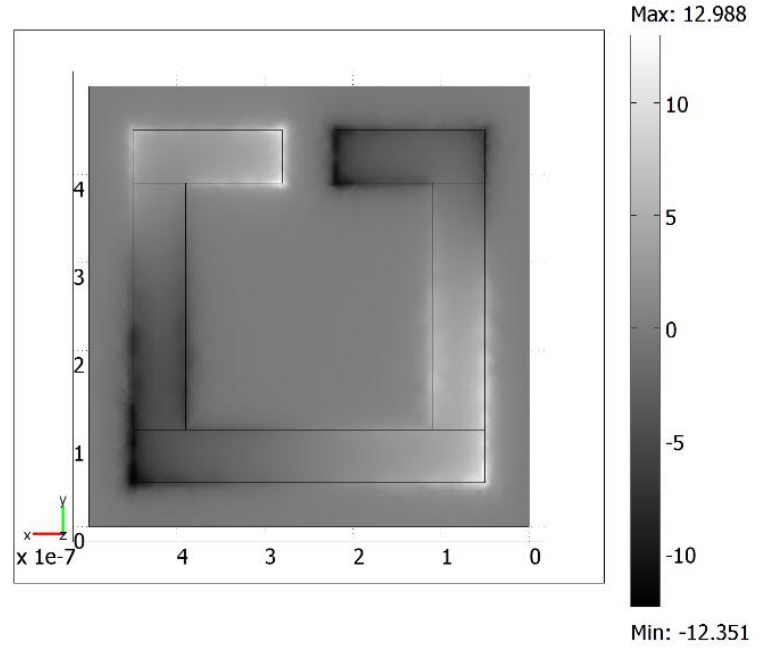

(b)

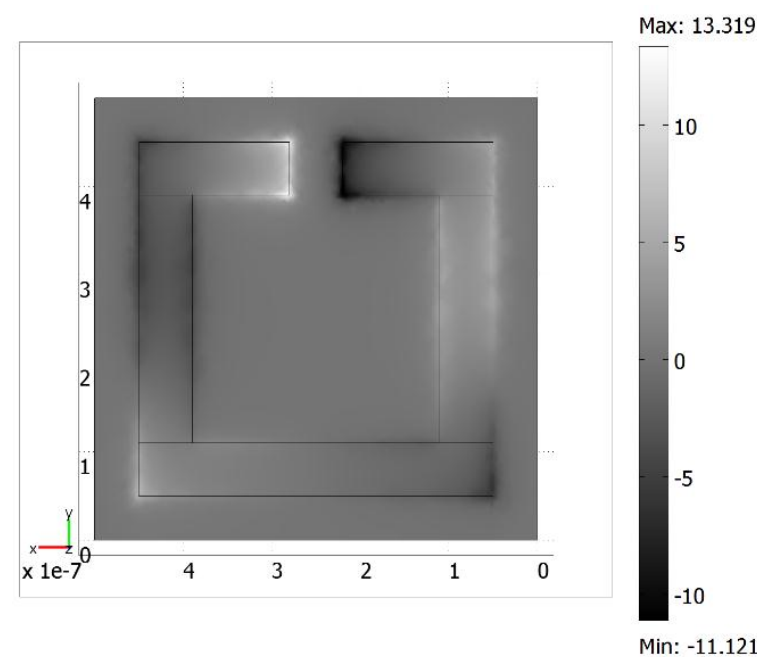

(c)

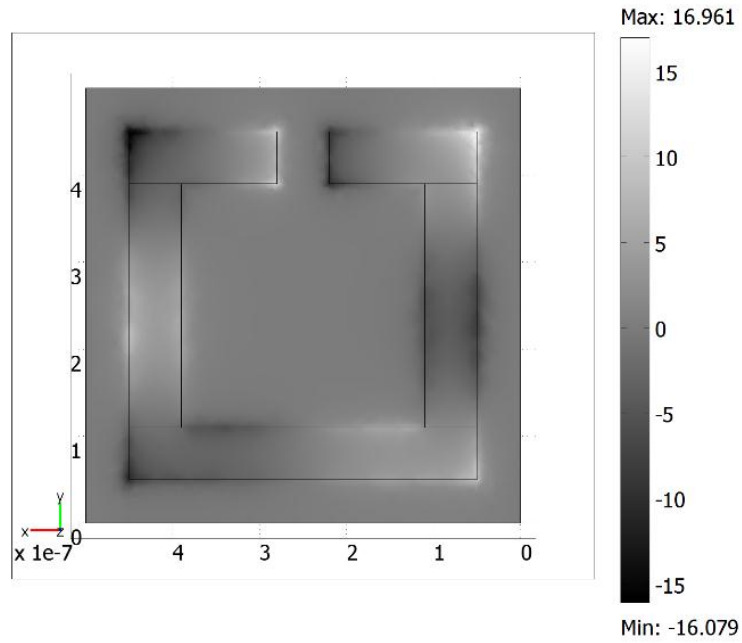

(d)

Fig. 3. Normalized electric field $\mathrm{z}$ component distribution for the four odd plasmonic modes.

For the applied electric field along the $\mathrm{y}$ direction three different resonances appear for the case $\mathrm{g}=280 \mathrm{~nm}$. According to the distribution of the normalized electric field $\mathrm{z}$ component, in the frequencies corresponding to the peaks (1) to (3) in Fig. 2(b), resonances correspond to the even plasmonic modes (Figures 4). For this polarization direction, due to the symmetry of the structure with respect to the polarization direction, electric fields in the legs parallel to the polarization direction have equal phase. 


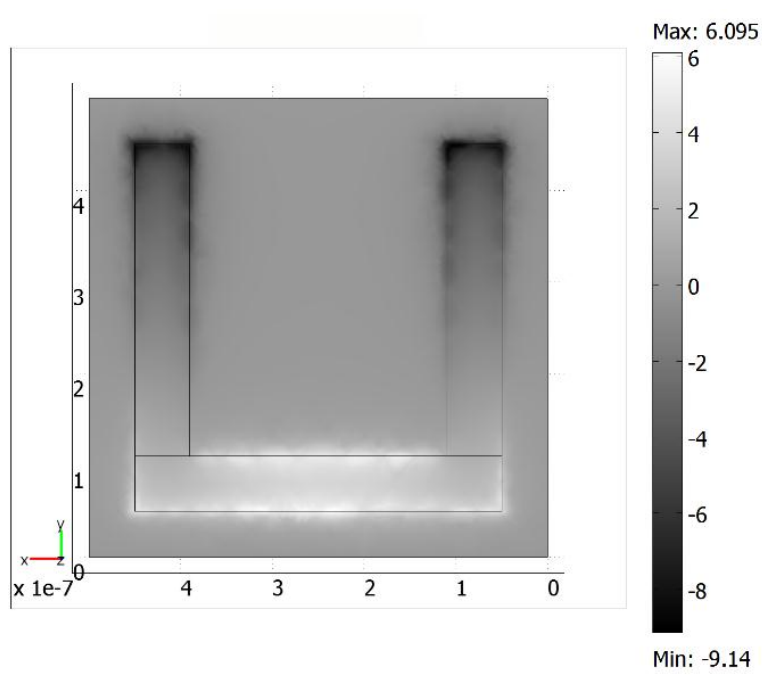

(a)

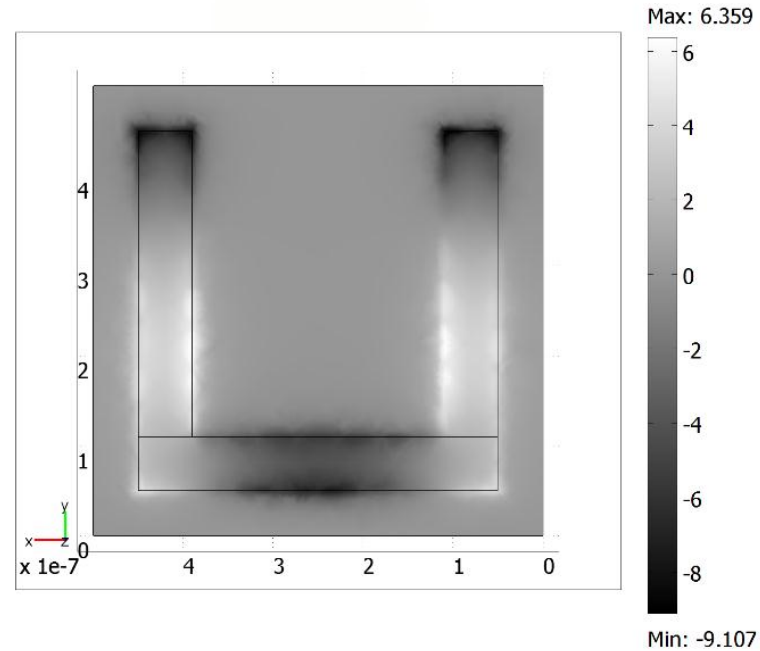

(b)

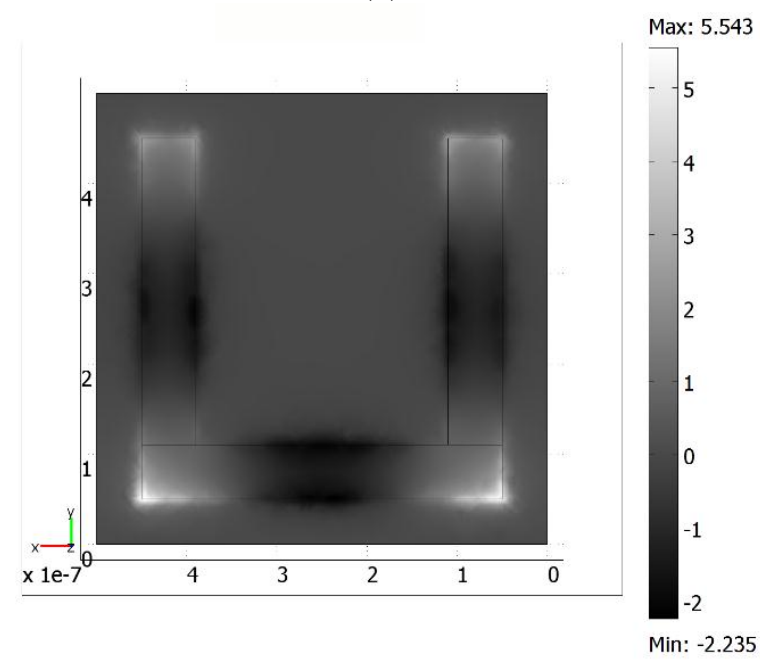

(c)

Fig. 4. Normalized electric field $\mathrm{z}$ component distribution for three even plasmonic modes.

Adopting the standing-wave model for surface Plasmon resonances[11], in order to obtain resonance and so larger LF in the wavelength $1.55 \mu \mathrm{m}$, the other geometrical parameters, $l_{x}$ and $l_{y}$, are decreased. Since the obtained LFs, around this specific wavelength, for the even modes are considerably smaller then than the odd modes, in the following just LF for the electric field in the $\mathrm{x}$ direction is represented. As seen in the Fig. 5, by reducing the $l_{x}$ amount, frequency of the first odd mode slightly shifts to the higher ones and LF becomes larger.

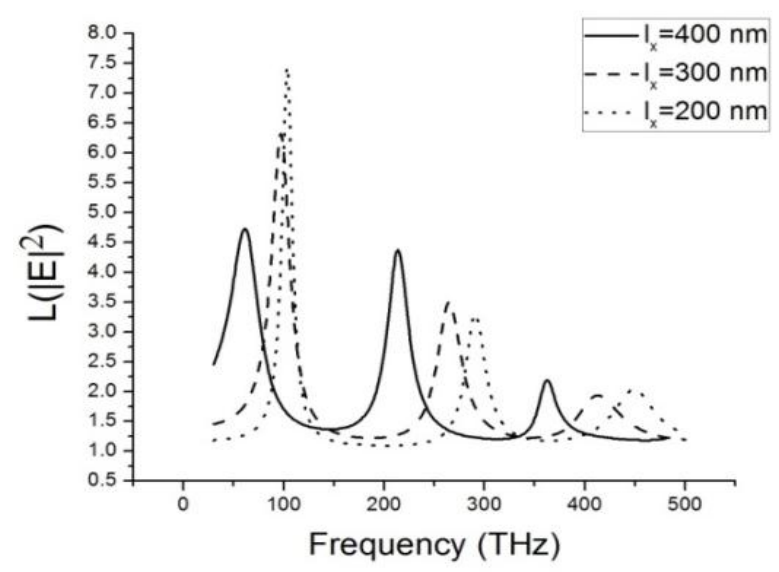

Fig. 5. Second-order LF for different lx amounts.

Considering the mentioned model, further increase of the resonance frequency can be possible by reducing the $l_{y}$ amount. As shown in the Fig. 6, reduction of the $l_{y}$ is accompanied by considerable blue shift of the resonance frequency, because the entire length of the SRR decreases more effectively.

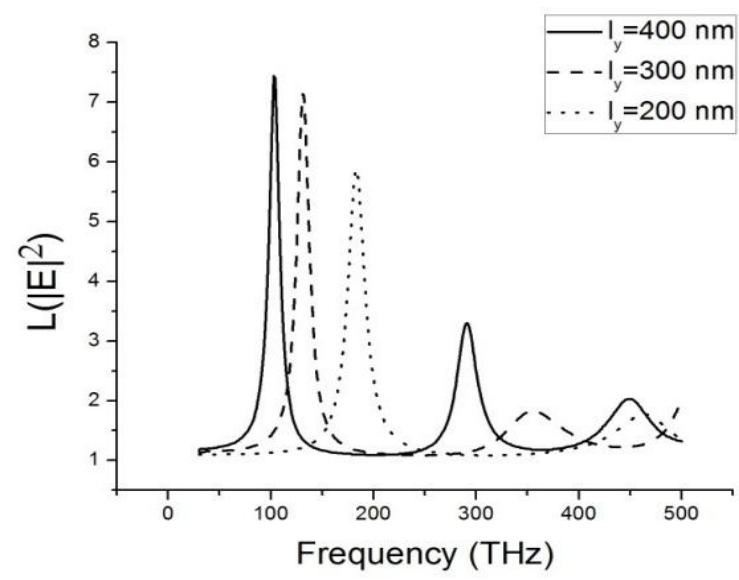

Fig. 6. Second-order LF for different $l_{y}$ amounts.

It is also clear that for the $l_{y}=200 \mathrm{~nm}$ resonance occurs in the frequency $193 \mathrm{THz}$ (corresponding to the wavelength $1.55 \mu \mathrm{m}$ ), but LF is smaller than the other cases since the 
filling factor of the metallic inclusions is reduced in the fixed unit cell size. So, the unit cell size is reduced to obtain larger LF. Figure 7 illustrates the LF for different unit cell sizes.

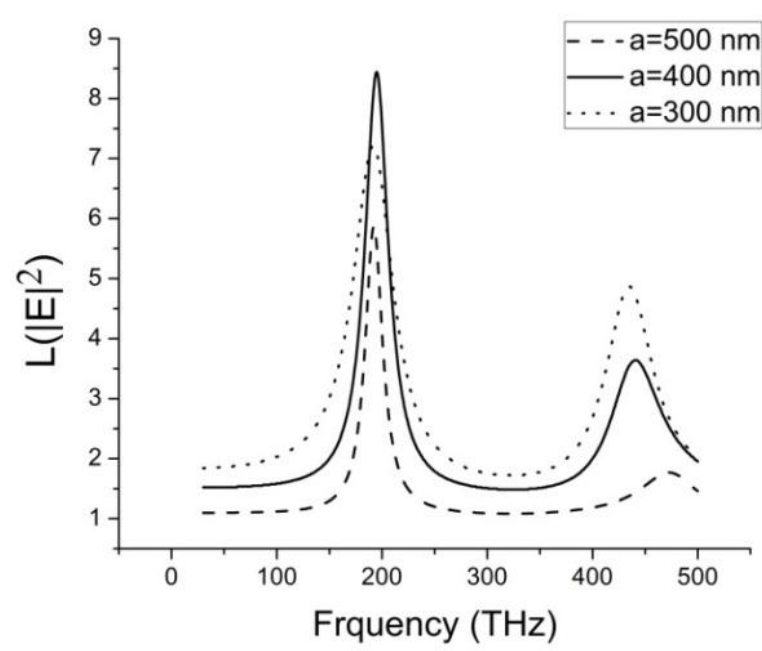

Fig. 7. Influence of the unit cell size on second-order LF.

When the unit cell size is decreased, resonance frequency remains invariant and maximum LF for the first odd mode in the frequency $193 \mathrm{THz}$ is obtained for $a=400 \mathrm{~nm}$. Reduction of the LF for more decreased unit cell sizes can be due to the diminished volume in which electric field is localized. Therefore, the geometrical parameters for the remaining investigations are $a=400 \mathrm{~nm}, l_{x}=l_{y}=200 \mathrm{~nm}$ which lead to the considerably large $\mathrm{LF}=8.5$ in the desired wavelength.

Afterward, the mentioned structure is considered to be embedded in a nonlinear dielectric. To show the enhancement of the nonlinear response of this structure quantitatively, the effective nonlinear susceptibility must be retrieved using the nonlinear retrieval method[24]. Based on this method, first the linear effective parameters (effective electric permittivity, $\varepsilon_{\text {eff }}$, and magnetic permeability, $\mu_{\text {eff }}$ ) should be determined. Here, the standard retrieval method is used to determine the effective linear parameters from the complex S-parameters (reflection and transmission coefficients)[30]. Complex S-parameters are obtained using the finite element frequency domain simulation of the considered structure. The real and imaginary parts of these effective parameters are illustrated in Fig. 8.

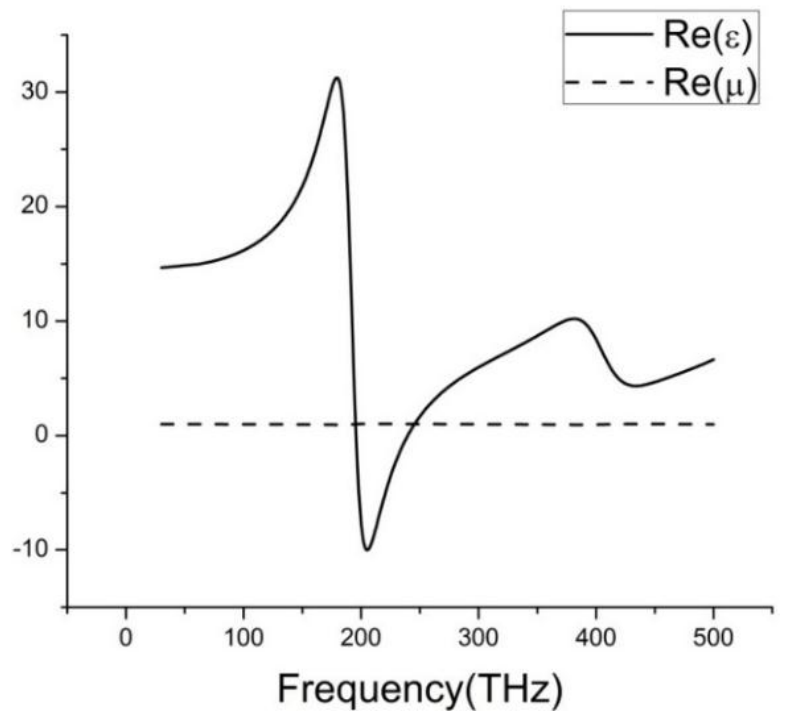

(a)

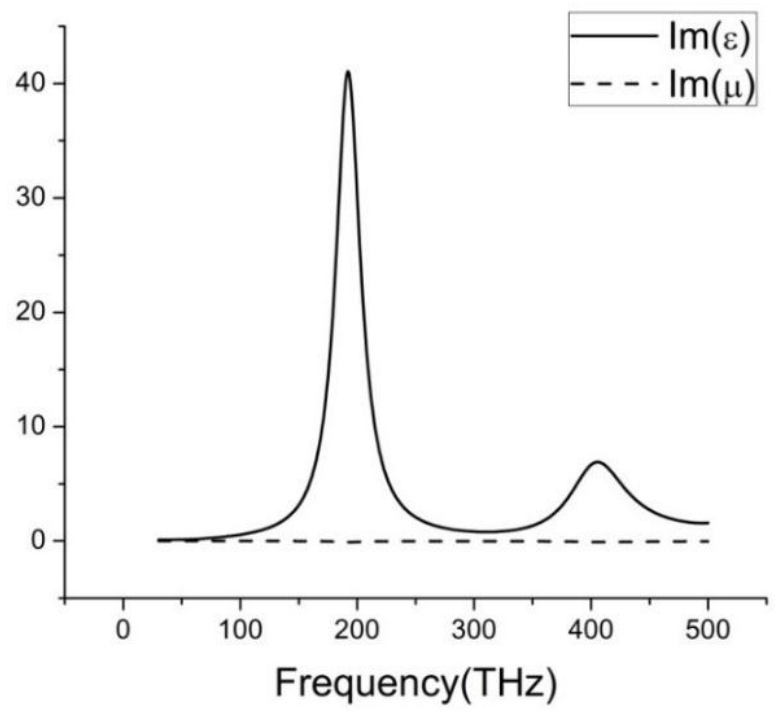

(b)

Fig. 8. Real (a) and imaginary (b) parts of the retrieved effective permittivity and permeability.

It is clear that the effective permittivity is more affected and appearance of a Lorentzian form change near the resonances confirms the plasmonic nature of these resonances.

Hereafter, to simulate the U-shaped structure with mentioned dimensions embedded in nonlinear dielectric, finite element time domain simulations are performed using the section "transient analysis of the electromagnetic waves" of the software. A time varying plane wave, as mentioned in the section (2), was 
applied to the structure and the obtained reflected and transmitted waveforms were Fourier transformed, which provides complex values of the amplitude. The amplitude of the Fourier transform of the transmitted signal has two peaks at fundamental frequencies and second harmonic (Fig. 9). The phase difference between transmitted and reflected signals with respect to the applied signal is also obtained.

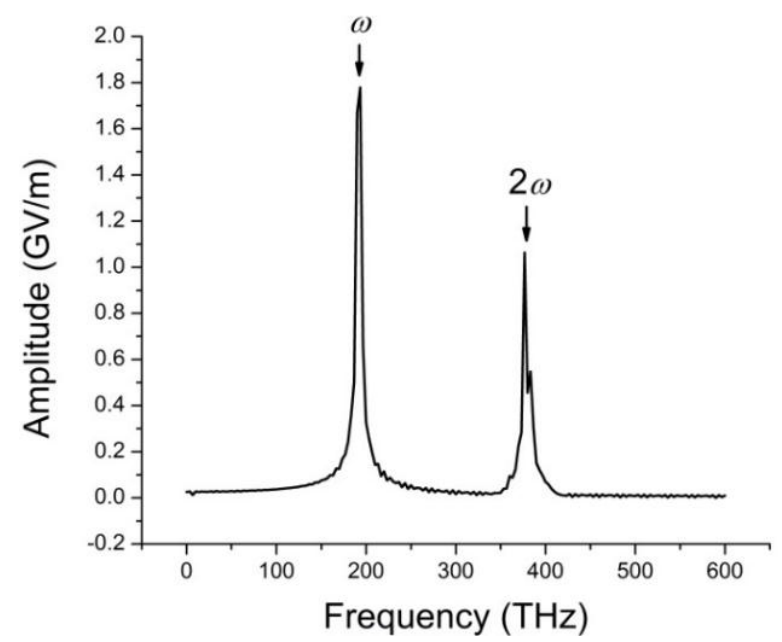

Fig. 9. Amplitude of the transmitted signals from the unit cell of the U-shaped nano-structure embedded in second order nonlinear dielectric, obtained using Fourier transforms.

According to these results, the retrieved effective nonlinear susceptibility is obtained to be more than 400 times larger than that of the nonlinear dielectric alone.

Efficiency of the SHG in the mentioned structure, considering the losses in resonance frequency, should also be estimated[31]. This efficiency is obtained to be more than 30 times larger than that of the nonlinear dielectric alone.

\section{IV.CONCLUSION}

In this article detailed investigation of the spectral response of the U-shaped nanostructures illustrate notable adjustment of the resonance frequencies and LF by varying the geometrical parameters and polarization directions. For the electric field parallel to the gap (x direction), four different odd modes are recognized for the gap size $60 \mathrm{~nm}$. Three even plasmonic modes are also excited for the gap size $280 \mathrm{~nm}$ when the electric field is parallel to the $y$ direction. Calculation of the LF for all structures reveals that this parameter in the resonance frequencies is larger for the odd modes compared to the even ones. Varying the geometrical parameters of the metallic structure which reduces the entire length of the U-shaped nano-structure leads to the blue shift of the resonance frequencies. Eventually, maximum LF in the wavelength $1.55 \mu \mathrm{m}$ is obtained to be 8.5 for the first odd plasmonic mode in specific geometrical parameters. Afterward, this nanostructure is considered to be embedded in nonlinear dielectric. Using the results of the finite element time domain simulations complex amplitude of the SHG is obtained. Via the nonlinear retrieval method, effective second-order susceptibility is calculated. Results of this step of our investigations indicate enhancement of the effective susceptibility two orders of magnitude for the SHG, compared to the considered nonlinear dielectric. Efficiency of the SHG from the considered nonlinear nano-structure is obtained to be more than 30 times compared to that of the nonlinear dielectric alone. So, this study reveals applicability of the considered structure as efficient frequency doublers or light source in nano-scale.

\section{ACKNOWLEDGMENT}

The authors would like to acknowledge Research Affairs Management of University of Tabriz for continuous and great support of this work.

\section{REFERENCES}

[1] N. Katsarakis, T. Koschny, M. Kafesaki, E.N. Economou, and C.M. Soukoulis, "Electric coupling to the magnetic resonance of split ring resonators," Appl. Phys. Lett. Vol. 84, no. 15, pp. 2943-2945, 2004.

[2] S. Linden, "Magnetic response of metamaterials at 100 terahertz," Science 80, Vol. 306, no. 5700, pp. 1351-1353, 2004.

[3] N. Katsarakis, G. Konstantinidis, A. Kostopoulos, R.S. Penciu, T. F. Gundogdu, M. Kafesaki, and E.N. Economou, "Magnetic response of split-ring resonators in the farinfrared frequency regime," Opt. Lett. Vol. 30, no. 11, pp. 1348-1350, 2005. 
[4] C. Enkrich, F. Perez-Willard, D. Gerthsen, J. Zhou, T. Koschny, C.M. Soukoulis, M. Wegener, and S. Linden, "Focused-ion-beam nanofabrication of near-infrared magnetic metamaterials," Adv. Mater. Vol. 17, no. 21, pp. 2547-2549, 2005.

[5] C. Enkrich, M. Wegener, S. Linden, S. Burger, L. Zschiedrich, F. Schmidt, J.F. Zhou, Th. Koschny, and C.M. Soukoulis, "Magnetic metamaterials at telecommunication and visible frequencies," Phys. Rev. Lett. Vol. 95, no. 20, pp. 5-8, 2005.

[6] A. K. Sarychev, G. Shvets, and V. M. Shalaev, "Magnetic plasmon resonance," Phys. Rev. E Stat. Nonlinear, Soft Matter Phys. Vol. 73, no. 3, pp. 1-10, 2006.

[7] D.R. Smith, W.J. Padilla, D.C. Vier, S.C. Nemat-Nasser, and S. Schultz, "Composite medium with simultaneously negative permeability and permittivity," Phys. Rev. Lett. Vol. 84, no. 18, pp. 4184 (1-4), 2000.

[8] M.W. Klein, C. Enkrich, M. Wegener, C.M. Soukoulis, and S. Linden, "Single-slit splitring resonators at optical frequencies: limits of size scaling.," Opt. Lett. Vol. 31, no. 9, pp. 1259-1261, 2006.

[9] C. Rockstuhl, T. Zentgraf, H. Guo, N. Liu, C. Etrich, I. Loa, K. Syassen, J. Kuhl, F. Lederer, and H. Giessen, "Resonances of split-ring resonator metamaterials in the near infrared," Appl. Phys. B Lasers Opt. Vol. 84, no. 1-2, pp. 219-227, 2006.

[10] C. Rockstuhl, F. Lederer, C. Etrich, T. Zentgraf, J. Kuhl, and H. Giessen, "On the reinterpretation of resonances in split-ringresonators at normal incidence," Opt. Express, Vol. 14, no. 19, pp. 8827-8836, 2006.

[11] F.J. Rodríguez-Fortuño, C. García-Meca, R. Ortuño, J. Martí, and A. Martínez, "Modeling high-order plasmon resonances of a U-shaped nanowire used to build a negative-index metamaterial," Phys. Rev. B - Condens. Matter Mater. Phys. Vol. 79, no. 7, pp. 1-10, 2009.

[12] V.K. Valev, A.V. Silhanek, B.D. Clercq, W. Gillijns, Y. Jeyaram, X. Zheng, V. Volskiy, O. A Aktsipetrov, G.A. E. Vandenbosch, M. Ameloot, V.V. Moshchalkov, and Th. Verbiest, "U-shaped switches for optical information processing at the nanoscale," Small, Vol. 7, no. 18, pp. 2573-2576, 2011.
[13] M. Lapine, "New degrees of freedom in nonlinear metamaterials," Phys. Status Solidi B, Vol. 254, pp. 1600462 (1-12), 2017.

[14] M. Kauranen and A.V Zayats, "Nonlinear plasmonics," Nat. Photon. Vol. 6, no. 11, pp. 737-748, 2012.

[15] F. B. P. Niesler, N. Feth, S. Linden, and M. Wegener, "Second-harmonic optical spectroscopy on split-ring-resonator arrays," Opt. Lett. Vol. 36, no. 9, pp. 1533-1535, 2011.

[16] S. Kruk, M. Weismann, A. Y. Bykov, E. A. Mamonov, I. A. Kolmychek, T. Murzina, N. C. Panoiu, D. N. Neshev, and Y. S. Kivshar, "Enhanced Magnetic Second-Harmonic Generation from Resonant Metasurfaces," ACS Photon. Vol. 2, no. 8, pp. 1007-1012, 2015.

[17] W. Schaich, "Second harmonic generation by periodically-structured metal surfaces," Phys. Rev. B, Vol. 78, no. 19, pp. 195416 (1-8), 2008.

[18] T. Laroche, F.I. Baida, and D. Van Labeke, "Three-dimensional finite-difference timedomain study of enhanced second-harmonic generation at the end of a apertureless scanning near-field optical microscope metal tip," J. Opt. Soc. Am. B, Vol. 22, no. 5, pp. 10451051, 2005.

[19] M.W. Klein, M. Wegener, N. Feth, and S. Linden, "Experiments on second-and thirdharmonic generation from magnetic metamaterials," Opt. Express, Vol. 15, no. 8, pp. 5238-5247, 2007.

[20] N. Feth, S. Linden, M. W. Klein, M. Decker, F. B. P. Niesler, Y. Zeng, W. Hoyer, J. Liu, S. W. Koch, J. V. Moloney, and M. Wegener, "Second-harmonic generation from complementary split-ring resonators," Opt. Lett. Vol. 33, no. 17, pp. 1975-1977, 2008.

[21] M. W. Klein, C. Enkrich, M. Wegener, and S. Linden, "Second-Harmonic Generation from Magnetic Metamaterials," Science 80, Vol. 313, no. 5786, pp. 502-504, 2006.

[22] F.B. Niesler, N. Feth, S. Linden, M.W. Klein, M. Decker, Y. Zeng, W. Hoyer, J. Liu, S.W. Koch, J.V. Moloney, and M. Wegener, "Second-harmonic generation from split-ring resonators on a GaAs substrate," Opt. Lett. Vol. 34, no. 13, pp. 1997-1999, 2009.

[23] D. Bar-Lev and J. Scheuer, "Efficient second harmonic generation using nonlinear substrates 
patterned by nano-antenna arrays," Opt. Express, Vol. 21, no. 24, pp. 29165-29178, 2013.

[24] S. Larouche and D.R. Smith, "A retrieval method for nonlinear metamaterials," Opt. Commun. Vol. 283, no. 8, pp. 1621-1627, 2010.

[25] R.A. Sabet and H. Khoshsima, "Utilizing the plasmonic resonance to enhance three wave mixing effects in nano-scale cut-wire," J. Opt. Quantum Electron. Vol. 47, no. 10, pp. 33373347, 2015.

[26] A. Rose, S. Larouche, and D.R. Smith, "Quantitative study of the enhancement of bulk nonlinearities in metamaterials," Phys. Rev. A - At. Mol. Opt. Phys. Vol. 84, no. 5, pp.053805 (1-13), 2011.

[27] R. W. Boyd, Nonlinear optics, Academic press, 2003.

[28] C. Fietz and C.M. Soukoulis, "Finite element simulation of microphotonic lasing system," Opt. Express, Vol. 20, no. 10, pp. 1154811560, 2012.

[29] D.R. Smith, S. Schultz, P. Markoš, and C.M. Soukoulis, "Determination of effective permittivity and permeability of metamaterials from reflection and transmission coefficients," Phys. Rev. B, Vol. 65, pp. 195104 (1-5), 2002.

[30] D.R. Smith, D.C. Vier, T. Koschny, and C.M. Soukoulis, "Electromagnetic parameter retrieval from inhomogeneous metamaterials," Phys. Rev. E - Stat. Nonlinear, Soft Matter Phys. Vol. 71, no. 3, pp. 1-11, 2005.

[31] J. Guo, Y. Xiang, X. Dai, and S. Wen, "Enhanced nonlinearities in double-fishnet negative-index photonic metamaterials," Prog. Electromagn. Res. Vol. 136, pp. 269-282, 2013.

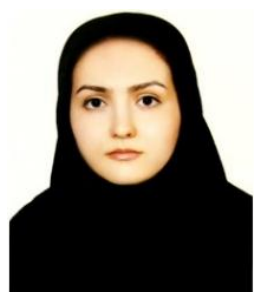

Rana Asgari Sabet was born in Tabriz, Iran, in 1984. She received the B.Sc. and M.Sc. degrees in atomic and molecular physics from the University of Tabriz, Tabriz, Iran, in 2007 and 2010, respectively, and the Ph.D. degree in Photonics from the University of Tabriz, Tabriz, Iran, in 2015. She has also been a Postdoctoral Researcher with University of Tabriz, Tabriz, Iran. Her main research activities are in the field of theoretical studies on linear and nonlinear metamaterials, plasmonics and nano-photonics and also experimental studies on liquid crystals.

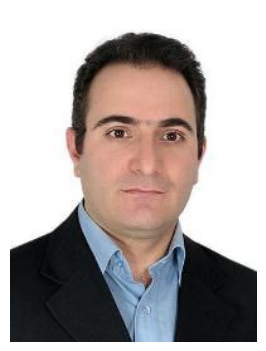

Habib Khoshsima was born in Tabriz, Iran, in 1970. He received the B.Sc. degree in atomic and molecular physics from the University of Tabriz, Tabriz, Iran, in 1983 and the M.Sc. and Ph.D. degrees in Laser Physics from the University of Tabriz, Tabriz, Iran, in 1997 and 2007, respectively. He is currently an Associate Professor at the Research Institute for Applied Physics and Astronomy. He has authored or coauthored more than 34 scientific papers in peerreferred international journals. His current researches are in the field of experimental study of the pure and doped liquid crystals and their applications and also theoretical study of the plasmonic structures, nano-antennas and plasmonic waveguides. 
THIS PAGE IS INTENTIONALLY LEFT BLANK. 